\title{
A Catenary System Analysis for Studying the Dynamic Characteristics of a High Speed Rail Pantograph
}

\author{
Tong-Jin Park, Byung-Jin Kim, Young-Yong Wang \\ Research assistant, Department of Precision Mechanical Engineering, Hanyang University, \\ Kyunggi-do 425-791, Korea \\ Chang-Soo Han* \\ Professor, Department of Mechanical Engineering, Hanyang University, \\ Kyunggi-do 425-791, Korea
}

\begin{abstract}
In this study, the dynamic response of a catenary system that supplies electrical power to high -speed trains is investigated. One of the important problems which is accompanied by increasing the speed of a high-speed rail, is the performance of stable current collection. Another problem which has been encountered, is maintaining continuous contact force between the catenary and the pantograph without loss of panhead. The dynamic analyses of the catenary based on the Finite Element Method (FEM) are performed to develop a pantograph suitable for high speed operation. The static deflection of the catenary, the stiffness variation in contact lines, the dynamic response of the catenary undergoing the force of a constantly moving load and the contact force were calculated. It was confirmed that a catenary model is necessary to study the dynamic characteristics of the pantograph system.
\end{abstract}

Key Words : Pantograph, Catenary, FEM (Finite Element Method), Contact Force, Contact Loss, High Speed Rail

\section{Nomenclature}

$m_{1} \quad$ : Mass of pantograph frame

$m_{2} \quad:$ Mass of pantograph plunger

$m_{3} \quad$ : Mass of pantograph panhead

$c_{1} \quad$ : Damping between body and pantograph frame

$c_{2}$ : Damping between pantograph frame and plunger

$c_{3} \quad$ : Damping between pantograph plunger and panhead

$k_{1} \quad:$ Stiffness between body and pantograph frame

$k_{2} \quad:$ Stiffness between pantograph frame and plunger

\footnotetext{
* Corresponding Author,

E-mail : cshan@ hanyang.ac.kr

TEL : + 82-31-400-5247; FAX : + 82-31-406-6242

Professor, Department of Mechanical Engineering, Hanyang University, Kyunggi-do 425-791, Korea. (Manuscript Received March 8, 2001; Revised February 22, 2002)
}

$k_{3} \quad:$ Stiffness between pantograph plunger and panhead

$F_{L} \quad:$ Static uplift force of pantograph

$F_{2}, F_{3} \quad$ : Aerodynamic uplift force

$m_{m}, m_{c}:$ Mass of a messenger wire element, contact wire element

$E I_{m}, E I_{c}:$ Bending stiffness of messenger wire, contact wire

$T_{m}, T_{c}$ : Tension of messenger wire, contact wire

$u_{m}, u_{c}$ : Displacement of messenger wire element, contact wire element

$k_{d}, k_{s}$ : Equivalent stiffness of dropper, support tower

$T \quad$ : Total kinetic energy

$V \quad$ : Total potential energy

$P \quad$ : Contact force 


\section{Introduction}

The high speed railway system that emerges as the next generation transportation system is characterized by high stability, high speed and passenger comfort, all of which are not part of other transportation systems. Various developed nations have put an emphasis on developing the technology. While Korea is concentrating its effort on assuring a basic technology and developing a speedier and more stable high speed railway.

One of accompanying problems to the high speed railway system is to ensure constant and stable current collection. For the stable operation of a railway, the catenary must be supplied with constant and stable electrical power through solid contact with the pantograph. If the velocity of the railway increases, the range of the catenary's dynamical variation will increase and then contact loss between the pantograph and the catenary might occur. In addition, there is going to be increased wear on the pantograph as electric shock and damage occurs. Research on the current collecting system's dynamic characteristics and a means to decrease dynamic variation is needed. Progress has been made in research for assuring the ability of high speed driving. Ockendon and Taylor (1971) described an approximate analytical formulation to determine contact force. Manabe (1994) conducted wave analysis research to study the response between the pantograph and the catenary with discrete support springs. Vinayagalingam (1983) studied contact force variation and panhead trajectory by using finite difference methods. Recently, an active pantograph was proposed for more stable current collection through maximizing the ability of the pantograph to follow the catenary. Connor, et al (1997) investigated the active pantograph with an optimal control algorithm. Resta, et al (1998) researched an active pantograph for a high speed rail system. For developing an active pantograph, it is important to estimate the behavior of the current collection system. These studies include the development of the controller which has an actuator at the base of the pantograph and feed- back signal of the displacement. However, there remains the difficult problem of applying a sensor to the pantograph conducting a high current.

As the driving speed of the KTX (Korea Train eXpress) is $350 \mathrm{~km} / \mathrm{h}$, it is necessary to investigate the feasibility of an active pantograph for better contact between the pantograph and the catenary. There have been many studies on the catenary system which determine the contact force with the pantograph system. Park, et al (1996) researched the caternary system using the FEM method based on the string modeling. Choi (1998) investigated the catenary system analytically for a high speed rail vehicle using a modal analysis. Wu and Brennan (1999) investigated the dynamic relation using the FEM between the catenary and the pantograph. These analyses on the catenary have been focused on the behavior of the catenary of the pantograph system of a high-speed rail to study contact loss.

In this paper, the dynamic characteristics of a catenary system using an FEM are analyzed to develop a suitable pantograph for a high speed rail system. By calculating the contact force which plays an important role in the dynamic characteristics of the pantograph, the stable current collecting ability of the pantograph can be determined. The contact force is analyzed in time domain by the dynamics of the catenary and the pantograph. At first, the catenary system of a high speed railway is assumed to be a continuous model. This model is analyzed by discrete-time domain modeling for the catenary system applying initial tensions at high-speed range. It is the analyzed using the FEM. To analyze the dynamic characteristics of the pantograph in time domain, the pantograph is assumed to be a 3 DOF linear mass-damper-spring system. Even though the pantograph has many parts, the parts of the pantograph can be regarded as the equivalent masses with installed springs and dampers. Even though the catenary is composed of an infinite length continuum model, it is modeled for 10 spans, over a total length of $630 \mathrm{~m}$ line. Dynamic characteristics such as contact force and displacement, etc. at a maximum velocity of $350 \mathrm{~km} / \mathrm{h}$ can be analyzed. 


\section{Modeling of the Catenary and the Pantograph}

There are three types of catenaries used in high speed rail way systems: simple, stitch and compound. The reason for having various catenaries is to unify the mechanical characteristics of contact wire contacting with the pantograph. In general, the compound catenary has lower stiffness variation than the simple catenary, and is better suited to a high speed rail system. The downside of the compound catenary is its high price ( $\mathrm{Wu}$, et al 1999). Hence, a simple catenary is usually adopted. The pantograph is divided into three main parts: a panhead, a plunger and a frame, all which are connected by springs and dampers. It is analyzed as a mass-spring-damper system with 3 DOF. This section describes modelings of the pantograph and the catenary.

\subsection{Composition of a Korean style high- speed railway catenary}

It is easier to ensure uniform elasticity with the compound catenary because of the small stiffness variation at the contact wire. The problem is that it costs a great deal to build. However, a simple catenary is showing its merits as being cost effective and easy to build, and it can attain sufficiently uniform elasticity. Hence, the simple catenary is being widely used especially in Europe and South Korea. Figure 1 shows the structure of the simple catenary system.

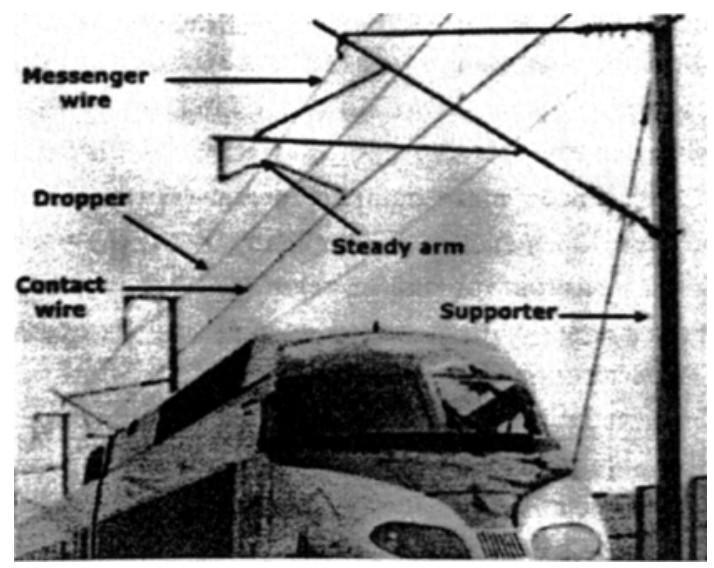

Fig. 1 The structure of simple catenary system
The contact wire is the part that supplies current through direct contact with the pantograph that is on the roof of the train. For mathematical modeling, a tensioning beam considering the high frequency response component is proposed. The messenger wire enables the contact wire to sustain a flexible distance from the track suspending supporter. In this paper, the messenger wire is assumed to be under the definite tension force of the contact wire. The dropper is the cable that spreads dynamic mass and connects the contact wire with the messenger wire to keep the contact wire from touching the messenger wire. The dropper is modeled as the mass and spring set with 9 pieces per $63 \mathrm{~m}$ span. The interval of each dropper is set at $4.5 \mathrm{~m}$ in the vicinity of the supporter and $6.75 \mathrm{~m}$ other places. The dropper plays an important role in providing uniform height and equal elasticity of the contact wire. The steady arm is the element that provides the stagger to prevent the one-sided wear on the contact wire through continuous contact between one point of the pantograph and the contact wire. Found at the supporter, the steady arm pushes and pulls the contact wire horizontally and is modeled as the mass and spring connected to the contact wire. The supporter that is a column supporting the messenger wire can be considered as a cantilever. Similarly, with the steady arm, the supporter can be modeled by the equivalent mass and spring. The space between two supports is called as a span, and generally, each support is set at intervals of $63 \mathrm{~m}$.

\subsection{A Finite element model of each part of the catenary}

Figure 2 shows the model for finite element analysis of the centenary.

In this paper, 10 spans with a total length of $630 \mathrm{~m}, 63 \mathrm{~m}$ per span are used. Because the object of this study is a high-speed railway having the velocity of $350 \mathrm{~km} / \mathrm{h}$, the composition of a 10 span catenary can be sufficient to study dynamic behavior not affected by boundary conditions. The KNR catenary condition that is being built now as the condition of the catenary in this study is used. Nine droppers are arranged per span and 


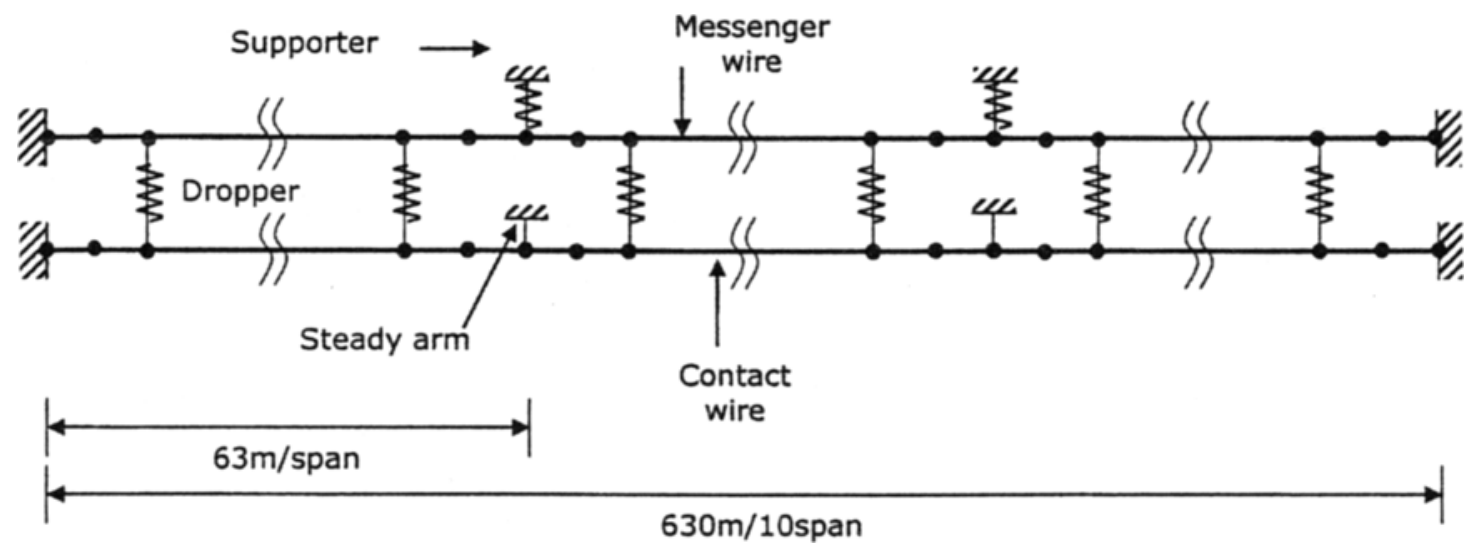

Fig. 2 Finite element model of a 10 span catenary

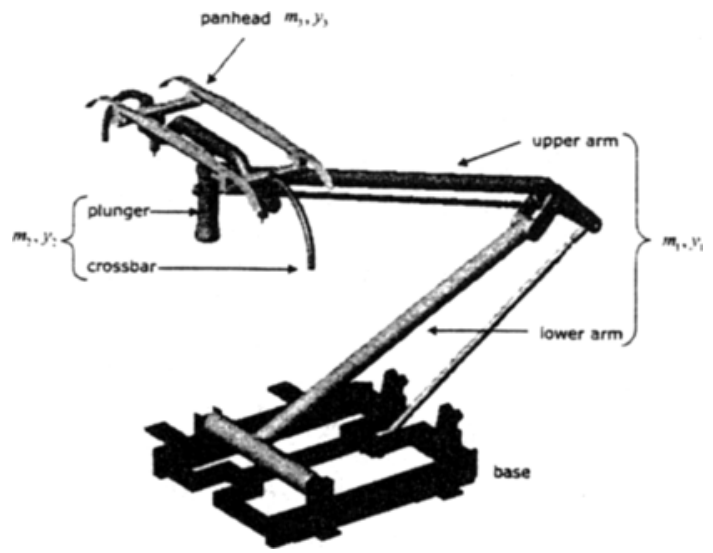

Fig. 3 GPU-type pantograph

the catenary is organized with boundary conditions that are fixed at both ends. The contact wire and the messenger wire are $1.4 \mathrm{~m}$ apart. While there is a little sagging, the model was simplified so that the initial condition was parallel and straight.

\subsection{The modeling of the pantograph system}

Causes of contact loss with the catenary system are wave propagation and elasticity irregularity, stiffness variation at dropper connection spots, unevenness at the connect surface of contact wire, mass variation at the part of the steady arm for support and connection, and variation of coach vibration and wind pressure from outside the train. For an uninterrupted and steady current supply, the design of a pantograph should have high following and quick reaction ability. It

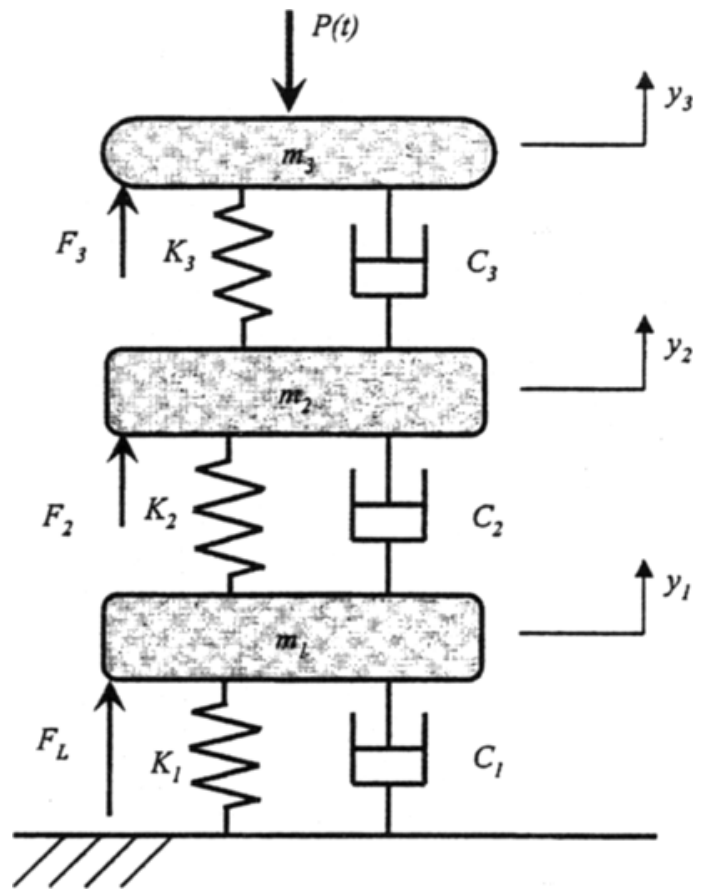

Fig. 4 Three DOF pantograph model

should also be insensitive to a variation of characteristic parameters. Figure 3 is the GPU type pantograph that is used in France. KTX will use a similar one.

It shows various parts of a pantograph. It is impossible to design a unifying model that can encompass all structures. However, a pantograph model is composed, like Fig. 4, using a highspeed railway with a 3-lumped mass, spring, damper, friction damper between each mass, and 
the structure that supplies external force for each mass, to analyze the general pantograph type similar to the one in Fig. 3.

\section{Analysis of the Catenary System and Pantograph}

The catenary system is modeled as a distributed parameter system and the pantograph is modeled as a lumped parameter system having masses and springs (Manabe, 1994, Park, et al, 1996). Generally, the catenary was modeled as a string using a FEM. However, $300 \mathrm{~km} / \mathrm{h}$ corresponds to $70 \%$ of the wave propagation speed and the analysis of the catenary using a string model is probably unreliable. In this study, the finite element analysis on the catenary using a beam model is accomplished to analyze the contact force at high speed. The catenary system should be discretized for analyzing a contact force with the pantograph. In this section, a lumped parameter system composed of catenary and pantograph is derived using Hamilton's Principle.

\subsection{Analysis of the catenary system}

The reason for not using a string model but using a beam model for the catenary is verified in this section. As stated in the introduction, the governing equations are derived from the catenary modeled with a tension beam to analyze high frequency responses. If $k$ is the wave number and $w$ the frequency, then derive the relation between and can be derived as;

$$
k= \pm\left\{-\frac{T}{2 E I}+\left[\left(\frac{T}{2 E I}\right)^{2}+\left(\frac{\rho}{E I}\right) w^{2}\right]^{1 / 2}\right\}^{1 / 2}
$$

In addition, because the wave propagation speed on the beam is $c=w / k$, then

$$
c= \pm\left\{\frac{T}{2 \rho}+\left[\left(\frac{T}{2 \rho}\right)^{2}+\left(\frac{E I}{\rho}\right) w^{2}\right]^{1 / 2}\right\}^{1 / 2}
$$

Equation (2) represents the wave propagation speed as the function of tension $T$ and bending stiffness $E I$. The above equation shows the characteristics varying with the wave frequency. Figure 5(a) shows the functional relation be- tween the wave propagation speed and tension $T$ when the bending stiffness is $E I C=322.2\left[\mathrm{Nm}^{2}\right]$. The wave propagation speeds of a string and of a beam in the low frequency range are almost the same. However, as the frequency increases, the wave propagation speed of the beam becomes higher than that of the string. As the bending stiffness of the beam decreases relative to the increased tension, the string model becomes similar to the beam model. Figure 5(b) represents the relation between the wave propagation speed and the bending stiffness when the tension $T=20,000$ [N].

As shown in Fig. 5(a), wave propagation speed increases more rapidly than the string model as the frequency increases. Dynamic characteristics of the tension beam have the property of the string and the beam depending on the relative magnitude of tension and bending stiffness. Tension has

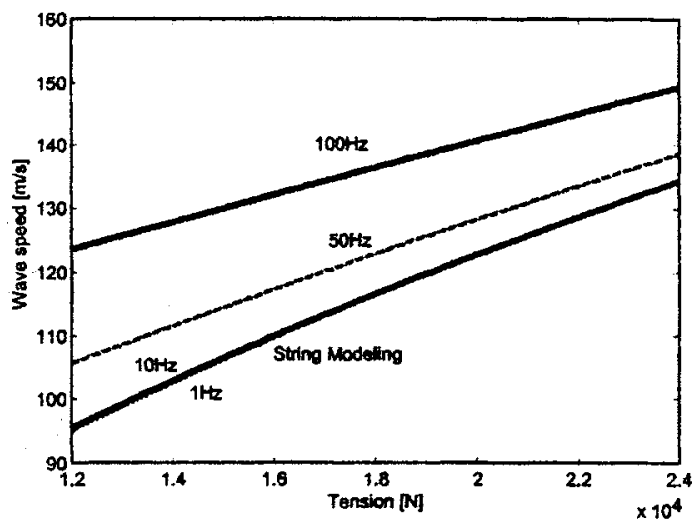

(a) Constant bending stiffness case

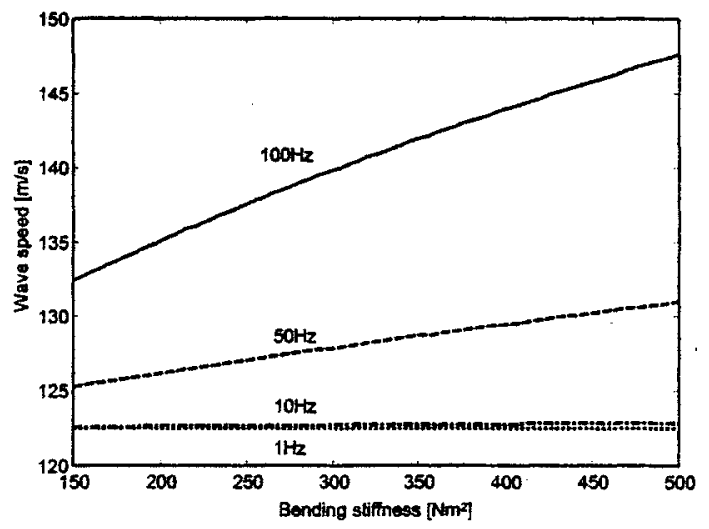

(b) Constant tension case

Fig. 5 Wave propagation velocity for a beam 
the primary effect on the real catenary except for the high frequency component. The effect of the bending stiffness must be considered in the high frequency at the speed of $350 \mathrm{~km} / \mathrm{h}$ of the KTX.

In this study, the catenary is analyzed as a tension beam with bending stiffness. The governing equation is derived using the energy variational method to accomplish a finite element analysis. The energy of each composed element on the catenary system can be shown as:

Kinetic energy

$$
\begin{aligned}
T= & \frac{1}{2} \int_{0}^{l} m\left(\frac{\partial u}{\partial t}\right)^{2} d x \\
= & \frac{1}{2}\left\{\int_{0}^{l}\left[m_{c}\left(\frac{\partial u_{c}}{\partial t}\right)^{2}\right] d x\right. \\
& \left.+\int_{0}^{l}\left[m_{m}\left(\frac{\partial u_{m}}{\partial t}\right)^{2}\right] d x\right\}
\end{aligned}
$$

Potential energy from the bending stiffness of the contact wire

$$
U_{E C}=\frac{1}{2} \int_{0}^{t} E I_{c}\left(\frac{\partial^{2} u_{c}}{\partial x^{2}}\right)^{2} d x
$$

Potential energy from the bending stiffness of the messenger wire

$$
\dot{U}_{E m}=\frac{1}{2} \int_{0}^{l} E I_{m}\left(\frac{\partial^{2} u_{m}}{\partial x^{2}}\right)^{2} d x
$$

Potential energy from tension of the contact wire

$$
U_{T c}=\frac{1}{2} \int_{0}^{i} T_{c}\left(\frac{\partial u_{c}}{\partial x}\right)^{2} d x
$$

Potential energy from bending stiffness of the contact wire

$$
U_{T m}=\frac{i}{2} \int_{0}^{l} T_{m}\left(\frac{\partial u_{m}}{\partial x}\right)^{2} d x
$$

Potential energy from droppers

$$
U_{D}=\frac{1}{2} \sum_{i=1}^{M} k_{d}\left\{u_{m}\left(x_{i}\right)-u_{c}\left(x_{i}\right)\right\}^{2}
$$

The potential energy from supporters

$$
U_{s}=\frac{1}{2} \sum_{i=1}^{M} k_{s}\left\{u_{m}\left(x_{i}\right)\right\}^{2}
$$

The total potential energy is the sum of all potential energy values.

$$
V=U_{T c}+U_{T m}+U_{E c}+U_{E m}+U_{D}+U_{S}
$$

Using the above energy related equation, the motion equation of the catenary can be obtained by applying Hamilton's Principle. First, the motion of the equation of the messenger wire can be expressed as:

$$
\begin{aligned}
m_{m} \frac{\partial^{2} u_{m}}{\partial t^{2}} & +\frac{\partial^{2}}{\partial x^{2}}\left(E I_{m} \frac{\partial^{2} u_{m}}{\partial x^{2}}\right)-\frac{\partial}{\partial x}\left(T_{m} \frac{\partial u_{m}}{\partial x}\right) \\
& +k_{d}\left(u_{m}-u_{c}\right) \delta\left(x-x_{n}\right) \\
& +k_{s} u_{m} \delta\left(x-\dot{x}_{s}\right)=0
\end{aligned}
$$

where, $m_{m}, T_{m}$, and $E I_{m}$ express unit mass of the messenger wire, tension force, and bending stiffriess respectively. Also, $k_{d}$ is the stiffness of the dropper, and $k_{s}$ is the equivalent stiffness of the supporter.

The motion of the equation of the contact wire can be expressed as;

$$
\begin{aligned}
m_{c} \frac{\partial^{2} u_{c}}{\partial t^{2}} & +\frac{\partial^{2}}{\partial x^{2}}\left(E I_{c} \frac{\partial^{2} u_{c}}{\partial x^{2}}\right)-\frac{\partial}{\partial x}\left(T_{c} \frac{\partial u_{c}}{\partial x}\right) \\
& -k_{d}\left(u_{m}-u_{c}\right) \delta\left(x-x_{n}\right)=P \delta(x-V t)
\end{aligned}
$$

where, $m_{c}, T_{c}$, and $E I_{c}$ express unit mass of the contact wire, tension force, and bending stiffness, respectively. $\delta$ is the delta function and $P$ is the contact force from the pantograph to the contact wire; which is the function of the vehicle velocity. Because the motion of the equation for the catenary is related to the contact force and because the contact wire and the messenger wires are connected to each other, the equations are difficult to solve analytically.

Therefore, in this paper, the displacement of the catenary is obtained based on the numerical analysis method using the FEM results in the absolute solutions on nodes and the approximate solutions between two nodes. The Newmark method was used for analyzing the catenary-FEM modeling in time domain, and the Full-Pivot method was used for calculating matrices.

\subsection{Finite element method}

To apply the finite element method, from Eqs. (11) and (12), the variation equations are derived as follows:

$$
\int_{0}^{L} w\left[m_{m} \frac{\partial^{2} u_{m}}{\partial t^{2}}+\frac{\partial^{2}}{\partial x^{2}}\left(E I_{m} \frac{\partial^{2} u_{m}}{\partial x^{2}}\right)\right.
$$




$$
\begin{gathered}
-\frac{\partial}{\partial x}\left(T_{m} \frac{\partial u_{m}}{\partial x}\right) \\
+k_{d}\left(u_{m}-u_{c}\right) \delta\left(x-x_{n}\right) \\
\left.+k_{s} u_{m} \delta\left(x-x_{s}\right)\right] d x=0 \\
\int_{0}^{L} w\left[m_{c} \frac{\partial^{2} u_{c}}{\partial t^{2}}+\frac{\partial^{2}}{\partial x^{2}}\left(E I_{c} \frac{\partial^{2} u_{c}}{\partial x^{2}}\right)\right. \\
-\frac{\partial}{\partial x}\left(T_{c} \frac{\partial u_{c}}{\partial x}\right) \\
-k_{d}\left(u_{m}-u_{c}\right) \delta\left(x-x_{n}\right) \\
-P \delta(x-V t)] d x=0
\end{gathered}
$$

where, $w$ is the weight function. The following equations can be derived by partial differentiations of Eqs. (13) and (14);

$$
\begin{aligned}
\int_{0}^{L} w m_{m} \frac{\partial^{2} u_{m}}{\partial t^{2}} d x & +\int_{0}^{L} E I_{m} \frac{\partial^{2} w}{\partial x^{2}} \frac{\partial^{2} u_{m}}{\partial x^{2}} d x \\
& +\int_{0}^{L} T_{m} \frac{\partial w}{\partial x} \frac{\partial u_{m}}{\partial x} d x \\
& +\int_{0}^{L} w\left(k_{d}\left(u_{m}-u_{c}\right) \delta\left(x-x_{n}\right)\right. \\
& \left.+k_{s} u_{m} \delta\left(x-x_{s}\right)\right) d x=0 \\
\int_{0}^{L} w m_{c} \frac{\partial^{2} u_{c}}{\partial t^{2}} d x & +\int_{0}^{L} E I_{c} \frac{\partial^{2} w}{\partial x^{2}} \frac{\partial^{2} u_{c}}{\partial x^{2}} d x \\
& +\int_{0}^{L} T_{c} \frac{\partial w}{\partial x} \frac{\partial u_{c}}{\partial x} d x \\
& +\int_{0}^{L} w\left(-k_{d}\left(u_{m}-u_{c}\right) \delta\left(x-x_{n}\right)\right) d x \\
= & \int_{0}^{L} P \delta(x-V t) d x
\end{aligned}
$$

Because both ends are considered to be free, the boundary conditions are zero shear force and zero moment. From Eqs. (15) and (16), displacement for each beam elements is obtained. Displacement and force of each node can be expressed as in Fig. 6 from a finite element of beam.

The beam is a long thin structural material that shows the bending effect clearer than the torsional effect or the axis direction effect. This bending effect is measured as vertical directional displace-

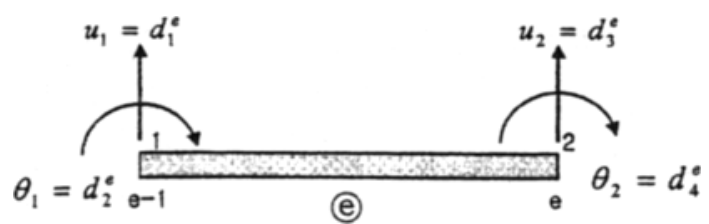

Fig. 6 Free diagram of a beam element ment and rotation. Therefore, there are 2 DOF per node for vertical directional displacement and rotation. In the case of the catenary, string tension force is applied with horizontal direction and the DOFs for horizontal direction are omitted. The reason for this is that the horizontal displacement of the catenary can be ignored.

The displacement at an arbitrary point can be expressed the shape function and DOF at each node, as follows;

$$
\begin{aligned}
& u_{h}(x)=\mathrm{N}^{\mathrm{T}} \mathrm{d}_{\mathrm{e}} \\
& w_{h}(x)=\mathrm{N}^{\mathrm{T}} \eta_{\mathrm{e}}=\eta_{\mathrm{e}}^{\mathrm{T}} \mathrm{N}
\end{aligned}
$$

where, $\mathbf{N}$ is the shape function, and $\eta$ is an arbitrary vector representing DOFs.

If Eq. (17) is inserted into Eqs. (15) and (16), $t$ can be expressed as a second ordinary differential equation;

$$
\mathbf{M} \ddot{\mathbf{d}}+\mathbf{K d}=\mathbf{F}
$$

where, matrix $\mathbf{M}$ is a mass matrix, and matrix $K$ is a stiffness matrix.

In Eq. (18), the stiffness matrix $K$ is composed of stiffness matrix $\mathbf{K}_{c}$ of the catenary and stiffness matrix $K_{d}$ of the dropper.

$$
\mathbf{K}=\mathbf{K}_{\mathbf{c}}+\mathbf{K}_{\mathbf{d}}
$$

where, $\mathbf{K}_{\mathbf{c}}$ is the diagonal matrix as;

$$
\mathbf{K}_{c}=\left[\begin{array}{llll}
\mathbf{K}_{e, \mathrm{ml}} & & \mathbf{0} \\
& \mathbf{K}_{\mathrm{e}, \mathrm{ct}} & & \\
& & \ddots & \\
\mathbf{0} & & \mathbf{K}_{e, \mathrm{cn}}
\end{array}\right]
$$

\subsection{Numerical analysis}

The algorithm for catenary system analysis is found in Fig. 7.

The analysis program is made up of the module that creates the stiffness matrix for the elements constituting a catenary, such as the beam, supporter, dropper, and the module that creates an element load vector applied to the catenary. This program includes assembling parts that bring the element matrices to form a global matrix and the dynamic analysis is performed by solving the differential equation per each time interval using the Newmark method, which makes the time integrating of a beam model stable. 


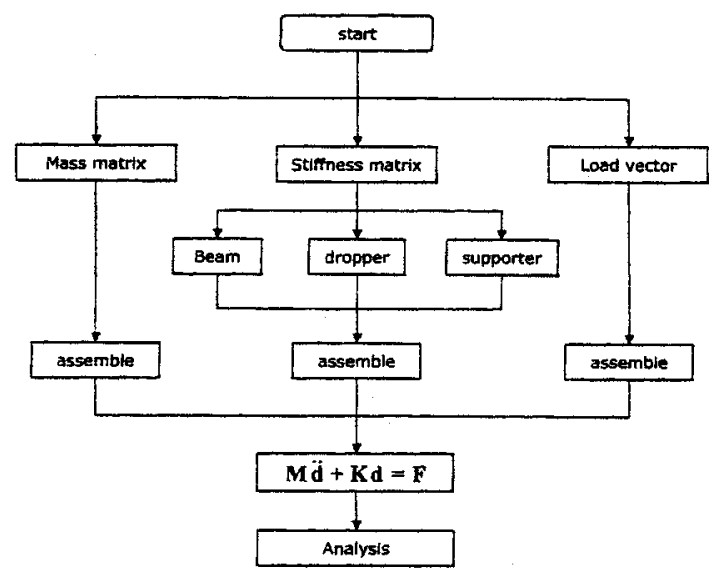

Fig. 7 Flow chart of FEM analysis

$\mathbf{M a}_{n+1}+\mathbf{K d} \mathbf{d}_{n+1}=\mathbf{F}\left(\mathrm{t}_{n+1}\right)$ at time $t=n+1$

The following relation is applied to this value.

$$
\begin{aligned}
\mathbf{d}_{n+1} & =\mathbf{d}_{n}+\Delta t \cdot \mathbf{v}_{n} \\
& +\frac{1}{2} \Delta t^{2}\left\{(1-2 \beta) \mathbf{a}_{n}+2 \beta \mathbf{a}_{n+1}\right\} \\
\mathbf{v}_{n+1} & =\mathbf{v}_{n}+\Delta t\left\{(1-\gamma) \mathbf{a}_{n}+\gamma \mathbf{a}_{n+1}\right\}
\end{aligned}
$$

where $\mathbf{d}_{n}, \mathbf{v}_{n}, \mathbf{a}_{n}$ are approximation of $\mathbf{d}\left(t_{n}\right)$, $\dot{\mathbf{d}}\left(t_{n}\right), \ddot{\mathbf{d}}\left(t_{n}\right)$

If Eq. (22) is inserted into Eq. (21), then Eq. (21) can be arranged about $\mathbf{a}_{n+1}, \beta$ and $\gamma$ determine stability and accuracy. As the system is the second order, $\beta$ and $\gamma$ are chosen to be $1 / 2$ and $1 /$ 4 respectively (Hughes, 1987). In the Newmark method, the stability is dependent on $\Delta t$. Generally, $\Delta t$ is determined by trial and error. In this paper, $\Delta t$ should be applied differently according to the speed. For instance, is chosen to be 0.027 at the speed of $300 \mathrm{~km} / \mathrm{h}$. Based on variations in the catenary condition, adjustment to the program is very simple. External force from the pantograph can be combined easily as a module in the program.

\subsection{The algorithm for calculation of contact force}

The 3 DOF pantograph ( $\mathrm{Wu}$, et at al 1999) model that is defined in Fig. 4 is included in this program. The equation of motion is derived at each nodal point of the 3 DOF model

$$
\begin{aligned}
& m_{3} \dot{y}_{3}-c_{3} \dot{y}_{2}-k_{3} y_{2}+c_{3} \dot{y}_{3}+k_{3} y_{3} \\
= & -P(t)+F_{3}
\end{aligned}
$$

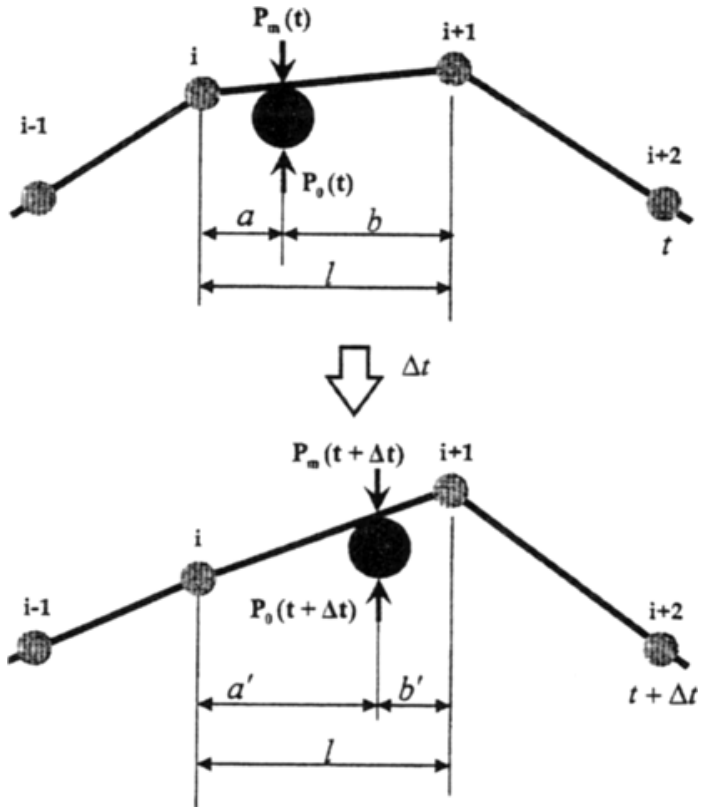

Fig. 8 The model of the contact force analysis

$$
\begin{aligned}
& m_{2} \ddot{y}_{2}-c_{2} \dot{y}_{1}-k_{2} y_{1}+\left(c_{2}+c_{3}\right) \dot{y}_{2}+\left(k_{2}+k_{3}\right) y_{2} \\
& \quad-c_{3} \dot{y}_{3}-k_{3} y_{3}=F_{2} \\
& m_{1} \dot{y}_{1}+\left(c_{1}+c_{2}\right) \dot{y}_{1}+\left(k_{1}+k_{2}\right) y_{1} \\
& \quad-c_{2} \dot{y}_{2}-k_{2} y_{2}=F_{L}
\end{aligned}
$$

The above equations of motion for the pantograph are included as a module in the catenary analysis program. The contact force that is expressed as $P(t)$ is supplied as an external force. It is most important to know the contact force between the contact wire and the panhead of the pantograph. In determining the magnitude of the contact force and the displacement of the contact wire at the contact point, the phenomenon that occurs during high speed driving can easily be understood. Based on the understanding of the high speed dynamics of the proposed simulation, the pantograph design values that show optimal dynamic characteristics can be set.

When the relationship between the contact wire and the contact distance from pantograph movement is assumed to be the same as in Fig. 8. The conditions below are added to solve the contact force.

$$
Y(t+\Delta t)=\gamma y_{i}(t+\Delta t)+\delta y_{i+1}(t+\Delta t)
$$

where, $\gamma=b(t+\Delta t) / l, \delta=a(t+\Delta t) / l$ 


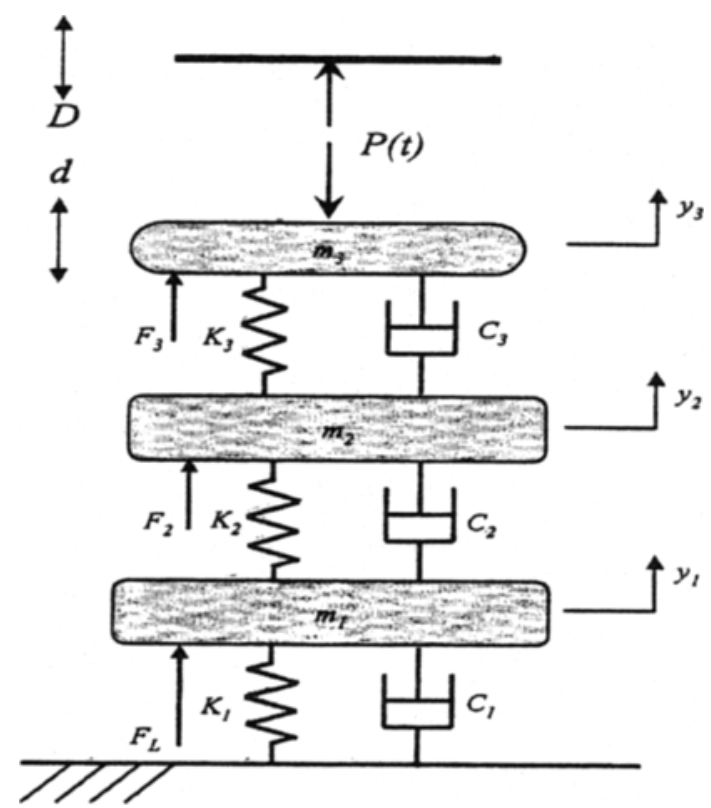

Fig. 9 Contact force between contact wire and pantograph

$F_{k}(t)$ that is the supplied force at each node point can be solved using the linear iteration method as follows;

$$
\begin{aligned}
F_{k}(t) & =\alpha P_{m}(t) & & (k=i) \\
& =\beta P_{m}(t) & & (k=i+1) \\
& =0 & & \text { (others) }
\end{aligned}
$$

where, $\alpha=b(t) / l, \beta=a(t) / l$

The algorithm for solving the contact force can be expressed as shown in Fig. 9:

In Fig. 9, the contact force occurs between the contact wire and the pantograph panhead. Because the contact force that is applied to the catenary system and pantograph system varies with time, the motion equation for the catenary and the pantograph systems must be solved at the same time. The iteration method is used for solving contact forces.

\section{Simulation}

The simulation for dynamic characteristic analysis, including a 3 DOF pantograph model is incorporated into the developed analysis program through contact force calculation algorithms. Contact force between the contact wires and the

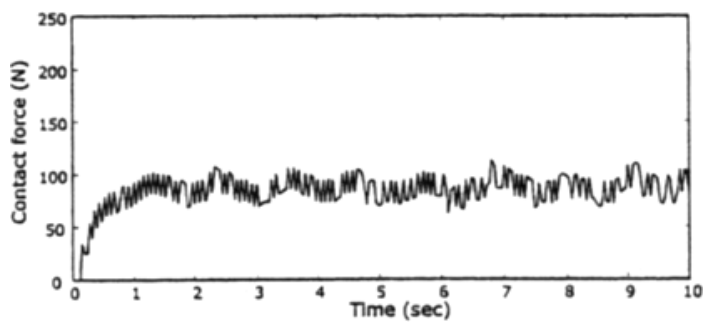

Fig. 10 Contact force of the contact wire $(v=200 \mathrm{~km} / \mathrm{h})$

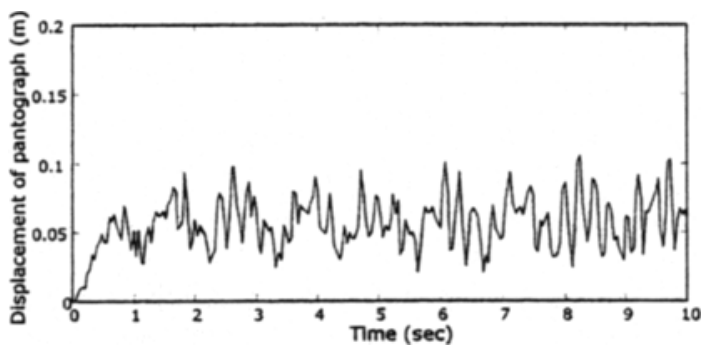

Fig. 11 Uplift displacements of the contact wire $(v=200 \mathrm{~km} / \mathrm{h})$

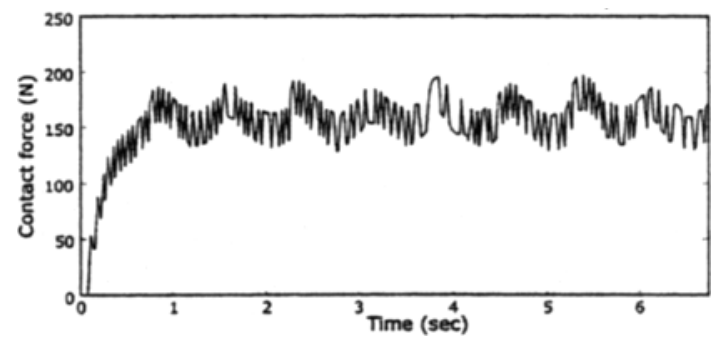

Fig. 12 Contact force of the contact wire $(v=250 \mathrm{~km} / \mathrm{h})$

pantograph are calculated for each velocity through the simulations. Figures 10 and 11 show contact force and displacement of the contact wire at a velocity of $200 \mathrm{~km} / \mathrm{h}$. The biggest contact force appeared at the supporter and the minimum contact force appeared at the center of the span.

Figures 12 and 13 show contact force and the displacement of the contact wire at $250 \mathrm{~km} / \mathrm{h}$.

In comparison with the $200 \mathrm{~km} / \mathrm{h}$ case, there is little difference in the shape of the result, but the average of the contact force and displacement show an increase in magnitude. Figures 14 and 15 show contact force and the displacement of the contact wire at $300 \mathrm{~km} / \mathrm{h}$. Similarly, Figs. 16 and 17 show these results at $350 \mathrm{~km} / \mathrm{h}$. 


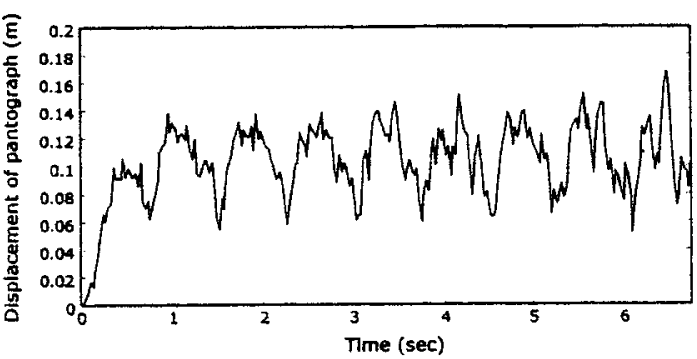

Fig. 13 Uplift displacements of the contact wire $(v=250 \mathrm{~km} / \mathrm{h})$

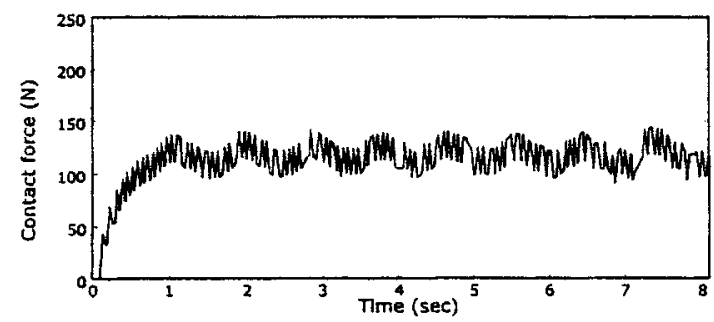

Fig. 14 Contact force of the contact wire $(v=300 \mathrm{~km} / \mathrm{h})$

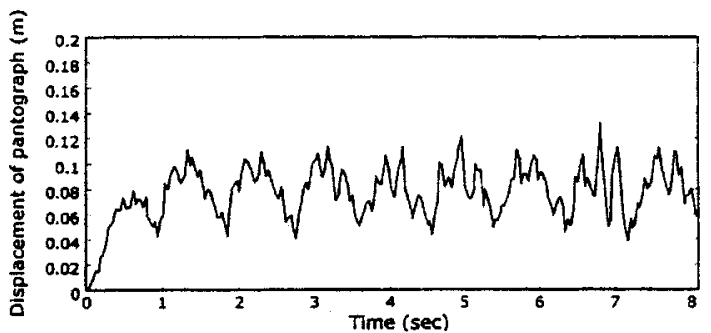

Fig. 15 Uplift displacements of the contact wire $(v=300 \mathrm{~km} / \mathrm{h})$

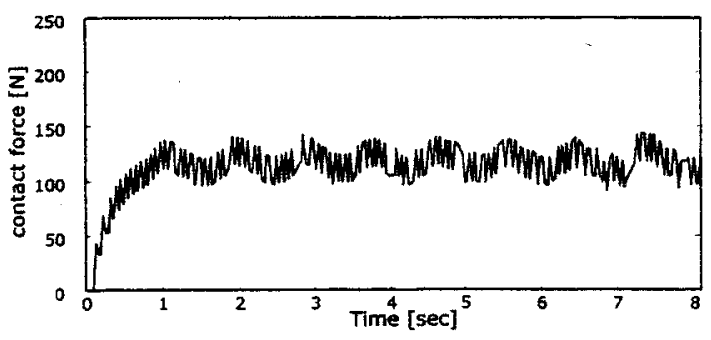

Fig. 16 Contact force of the contact wire $(v=350 \mathrm{~km} / \mathrm{h})$

Table 1 is arranged by the contact force and the displacement of the contact wire at each velocity. With velocities of $200,250,300$, and $350 \mathrm{~km} / \mathrm{h}$, the average contact force, displacement, and standard deviation are compared.
Table 1 Analysis results of the comparison of the contact force and the displacement

\begin{tabular}{c|r|r|c|c}
\hline \multirow{2}{*}{$\begin{array}{l}\text { Velocity } \\
{[\mathrm{Km} / \mathrm{h}]}\end{array}$} & \multicolumn{2}{|c|}{ Contact force $[\mathrm{N}]$} & \multicolumn{2}{c|}{ Displacement $[\mathrm{m}]$} \\
\cline { 2 - 5 } & Average & $\begin{array}{c}\text { Standard } \\
\text { Deviation }\end{array}$ & Average & $\begin{array}{c}\text { Standard } \\
\text { Deviation }\end{array}$ \\
\hline 200 & 83.33 & 16.94 & 0.0562 & 0.019 \\
\hline 250 & 112.85 & 22.43 & 0.0768 & 0.021 \\
\hline 300 & 153.22 & 29.68 & 0.1025 & 0.027 \\
\hline 350 & 192.93 & 37.23 & 0.1301 & 0.034 \\
\hline
\end{tabular}

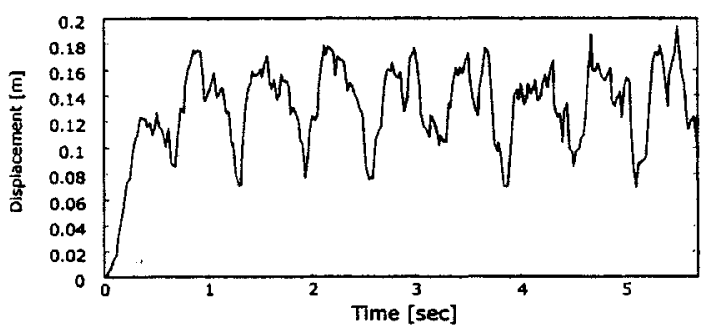

Fig. 17 Uplift displacements of the contact wire $(v=350 \mathrm{~km} / \mathrm{h})$

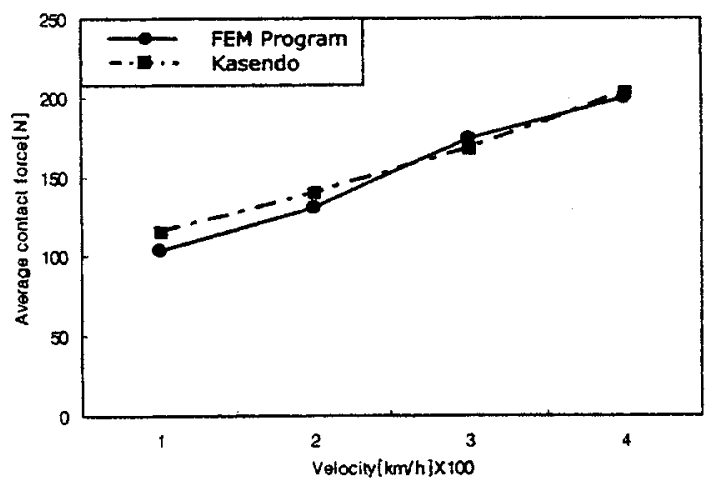

Fig. 18 Comparison with the Kasendo for the average contact force

The contact force at $350 \mathrm{~km} / \mathrm{h}$ is about twice that at $200 \mathrm{~km} / \mathrm{h}$. Deviation increased by more than $200 \%$. If the deviation of the contact force is increased, the possibility of contact loss would be extremely high and the performance of the current collection would be worse.

The program developed in this paper was compared with the 'Kasendo' program (the catenary analysis program developed in Japan) to prove its validity. It analyzes the finite element of a catenary modeled as a string. The average and standard deviation of the contact force is compared in Figs. 18, 19. 


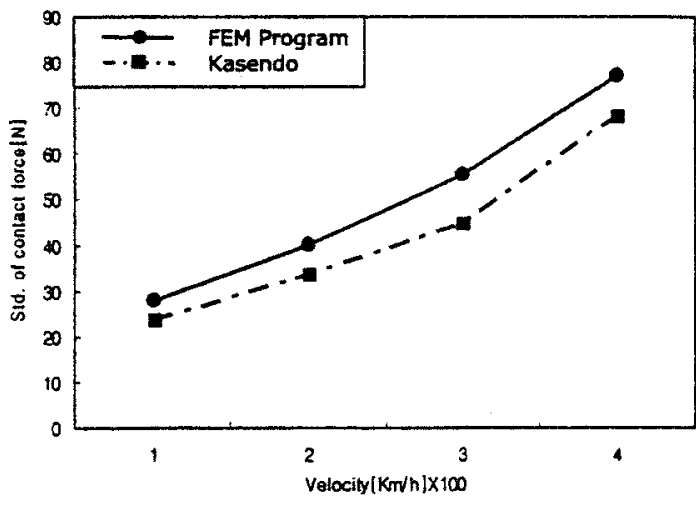

Fig. 19 Comparison with the Kasendo for the standard deviation of contact force

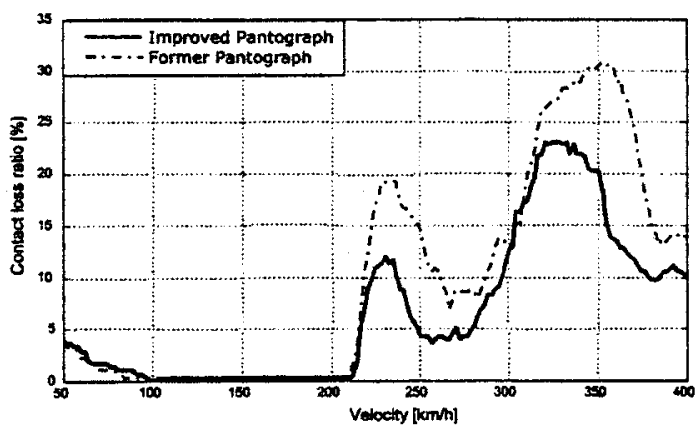

Fig. 20 Comparison of the contact loss ratio of the improved pantograph

The average value of the contact force is almost the same as is found the results of 'Kasendo', the guide to the analysis of the pantograph of the Shinkansen. The standard deviation of the contact force is higher than that of the Kasendo program. That difference came from the modeling between the string and the beam. As stated earlier, the more precise results of contact force are derived from the beam model than from the string model.

To improve the performance of the contact loss of the pantograph using the catenary analysis program developed in this paper, the design variables of the pantograph are modified. Figure 20 shows the reduction in the contact loss by improving the design variables of the pantograph.

In this paper, the contact loss is defined as contact loss time divided by the total operating time. The results in Fig. 20 show the possibility of decreasing the contact loss by modifying the design variables of the pantograph through the analysis program of the catenary and the pantograph.

\section{Conclusions}

In this paper, an analysis tool that analyzes dynamic characteristics of the pantograph at a high speed is developed. Even though there is the Japanese catenary analysis program, Kasendo, that has a similar function to this program, this program models the contact wire and messenger wires as a tensioning beam that has arbitrary bending stiffness and tension force. Therefore, it is believed that this program can be applied to the next generation of high speed railways having a target velocity of $350 \mathrm{~km} / \mathrm{h}$. A subject for future study is the development of a user friendly program which includes user interface and verification based on a real system experiment.

\section{References}

Balestrino A., Bruno O., Landi A. and Sani L., 2000, "Innovative Solutions for Overhead Catenary-Pantograph System : Wire Actuated Control and Observed Contact Force," Vehicle System Dynamic, Vol. 33, pp. 69 89.

Johnson C., 1995, Numerical Solution of Partial Differential Equations by the Finite Element Method, Cambridge.

Jensen C. N., True H. and Consult S., 1998, "Dynamic of an Electrical Overhead Line System and Moving Pantographs," Vehicle System Dynamic, Vol. 28, pp. 104 113.

O'Connor D. N., Eppinger S. D., Seering W. P. and Wormley D. N., 1997, "Active Control of a High-Speed Pantograph," Journal of Dynamic Systems, Measurement, and Control, Vol. 119, pp. $1 \sim 4$.

Diana G., Fossati F. and Resta F., 1998, "High Speed Railway: Collecting Pantographs Active Control and Overhead Lines Diagnostic Solutions," Vehicle System Dynamic, Vol. 30, pp. $69 \sim 84$.

Hughes, 1987, The Finite Element Method, Prentice Hall Inc.

Ockendon J. R. and Tayor, A. B., 1971, "The 
dynamics of a current collection system for an electric Locomotive," Proceedings of the Royal Society of London, Series A 211, pp. 336 - 357.

Manabe K., 1994, "Periodical Dynamic Stabilities of a Catenary-Pantograph System," $Q R$ of $R T R I$, Vol. 35, No. 2, pp. 112 117.

Chapra S. C. and Canale R. P., 1990, Numerical Method for Engineers, McGraw-Hill Thomas J. R.

Sukyung Park, Seamoon Kim, Yang-Hann Kim, 1996, “ Wave Propagation Characteristics along a Simple Catenary with Arbitrary Impedance Conditions," KSME Journal, No. 11, Vol. 20 , pp. $3463 \sim 3473$.

Wu T.X. and Brennan M. J., 1998, "Basic Analytical Study of Pantograph-catenary System Dynamics," Vehicle System Dynamic, Vol. 30, pp. $443 \sim 456$.

Wu T. X. and Brennan M. J., 1999, "Dynamic
Stiffness of a Railway Overhead Wire System and Its Effect on Pantograph-catenary System Dynamics," Journal of Sound and Vibration, Vol. 219 (3), pp. 483 502 .

Yagi T., Stensson A. and Hardell C., 1996, "Simulation and Visualization of the Dynamic Behavior of an Overhead Power System with Contact Breaking," Vehicle System Dynamic, Vol. 25, pp. 31 49.

Vinayagalingam T., 1983, "Computer evaluation of controlled pantographs for current collection from simple catenary overhead equipment at high speed," ASME Journal of Dynamics Systems Measurement and Control, Vol. 105, pp. 287 294.

Yeon-Sun Choi, 1998, "Analysis on the Design Parameters of Current Collection System of High Speed Train," KSME Journal, No. 6, Vol. 22, pp. $1002 \sim 1008$. 
Reproduced with permission of the copyright owner. Further reproduction prohibited without permission. 